\title{
Faster than the brain's speed of light: Retinocortical interactions differ in high frequency activity when processing darks and lights
}

\author{
Britta U. Westner ${ }^{1,2}{ }^{*}$ and Sarang S. Dalal ${ }^{2}$
}

\author{
1 Department of Psychology, University of Konstanz, Konstanz, Germany \\ 2 Center of Functionally Integrative Neuroscience, Department of Clinical Medicine, Aarhus \\ University, Aarhus, Denmark \\ * Correspondance to: britta.wstnr@gmail.com
}

\section{Abstract}

Some studies suggest that the processing of darks benefits from greater neural resources in the visual system and potentially occurs faster. However, evidence from the human is still sparse, especially with respect to retinocortical interactions. We recorded retinal and cortical responses to $480 \mathrm{~ms}$ light flashes simultaneously with electroretinography (ERG) and magnetencephalography (MEG) in ten participants and analyzed the high frequency responses to the flash onsets and offsets.

We show that high frequency oscillations for flash offsets occur earlier than flash onsets in the cortex but not in the retina. Interestingly, while the onset activity involved a wide range of frequencies $(55-195 \mathrm{~Hz}$ in the retina, and $55-145 \mathrm{~Hz}$ in the cortex), the offset response was restricted to the $75-95 \mathrm{~Hz}$ frequency band in both retina and cortex. The results suggest faster propagation times but not earlier retinal processing for darks than lights, suggesting a thalamic role. They also support previous findings that the retinal high frequency activity is transmitted to cortex. Furthermore, the outcomes add to the ongoing discussion about the function of narrowband oscillations in the human visual system. 


\section{Introduction}

In 1938, Hartline discovered that the processing of light increments and light decrements is done separately by two pathways in the retina, the ON- and OFF-pathways, which commence with sign-inverting and sing-conserving bipolar cells at the first synapse of the photoreceptors (Werblin and Dowling, 1969). These channels have long been treated as parallel, however, studies suggest functional and neuronal asymmetries in these pathways.

A behavioral advantage for the detection of dark objects or light decrements over light objects has been reported in several psychophysical studies (e.g., Blackwell, 1946; Krauskopf, 1980; Bowen et al., 1989; Chubb and Nam, 2000; Buchner and Baumgartner, 2007). More recently, Komban et al. (2011) reported faster and more accurate reactions for dark squares compared to light squares on a uniform binary noise background at suprathreshold but not threshold levels. This advantage for dark stimuli, however, vanished if the binary noise background was corrected for the irradiation illusion, which is the effect that light objects on a dark background seem larger than their dark counterparts (Galilei, 1632; von Helmholtz, 1867). These results raise the question at which stage of the visual system do potential functional asymmetries in the $\mathrm{ON}$ and $\mathrm{OFF}$ pathway emerge - and what precisely are the neural underpinnings of these often reported behavioral advantages of darks over lights.

In visual cortex, responses to light decrements are found to be stronger than responses to light increments in both electroencephalography (EEG) and functional magnetic resonance imaging (fMRI) recordings (Zemon et al., 1988; Zemon et al., 1995; Olman et al., 2008). Multiunit recordings from cat visual cortex show faster response latencies (defined as $40 \%$ of maximum response) in OFF-dominated cortical sites (Komban et al., 2014). The number of geniculate afferents at the representation of the area centralis in cat visual cortex is higher in the OFF-pathway (Jin et al., 2008). Otherwise, Yeh et al. (2009) reported more black-dominant neurons in layers 2 and 3 of primary visual cortex (V1) of macaque monkeys, but a balanced amount of black- and white-dominant neurons in the thalamic input layer $4 \mathrm{c}$ of visual cortex and thus con- 
clude that advantages for the processing of darks are generated or at least amplified in the visual cortex and not the thalamus. Subsequently, they showed that the neural circuitry in V1 seems to enhance responses to darks: they found a temporal advantage in the processing of darks in the thalamic input layer $4 \mathrm{c}$, but not in the later stages of cortical visual processing (Xing et al., 2010). A potential advantage for dark stimuli at the thalamic level is further supported by a study of Jin et al. (2011), which reported faster processing for light decrements than increments in the lateral geniculate nucleus (LGN) of the cat thalamus. Thus, studies which focused on cortical and thalamic processing of darks and lights suggest that there are greater neural resources for darks, however, they do not agree at which stage of the visual system these advantages are introduced.

At the retinal stage, evidence for functional asymmetries in the ON- and OFF-pathways is mixed. While some studies find no asymmetries at all (Kremers et al., 1993; Benardete and Kaplan, 1997; Benardete and Kaplan, 1999), others do report differences in $\mathrm{ON}$ and $\mathrm{OFF}$ processing. For example, it has been shown that OFF bipolar cells outnumber ON bipolar cells in the central retina by twofold (Ahmad et al., 2003). OFF ganglion cells seem to have narrower dendritic and thus narrower receptive fields than their ON counterparts (Wässle et al., 1981; Morigiwa et al., 1989; Dacey and Petersen, 1992; DeVries and Baylor, 1997), which show more overlap than ON dendritic fields (Borghuis et al., 2008). This suggests that more resources are allocated to the OFF pathway (Balasubramanian and Sterling, 2009). Furthermore, OFF neurons respond fairly linear with light decrements, whereas ON neurons reveal a pronounced non-linearity and saturate their responses even with small increases in luminance (Chichilnisky and Kalmar, 2002; Kremkow et al., 2014). It has also been shown, however, that OFF cell currents are rectified by ON cells (Zaghloul et al., 2003; Liang and Freed, 2010). Regarding the response kinetics of ON and OFF retinal cells, it has been hypothesized that OFF bipolar cells are faster, since no biochemical sign inversion of the light response is needed - in contrast to ON bipolar cells (Nawy and Jahr, 1990; Chichilnisky and Kalmar, 2002). Indeed, the initial response (defined as $5 \%$ of maximum response) was shown to be slightly faster for OFF-bipolar 
cells (Chichilnisky and Kalmar, 2002). This finding is strengthened by several studies which also show faster responses for light decrements in the retina (Copenhagen et al., 1983; Zaghloul et al., 2003; Burkhardt et al., 2007; Gollisch and Meister, 2008; Nichols et al., 2013). However, Chichilnisky and Kalmar (2002) reported this temporal advantage only for the initial response, whereas the time to peak was shorter for ON bipolar cells and not OFF bipolar cells (also see Lankheet et al., 1998).

In summary, while there is an evident advantage for darks over lights on the behavioral level, the potential functional asymmetries in the ON and OFF pathways throughout the visual system are less well understood and especially evidence from the human visual system is still sparse. In the present study, we aim at investigating the shape and timing of activity patterns elicited by flash onsets and offsets in the human visual system by recording retinal and cortical responses simultaneously.

Retinal potentials and high frequency oscillations Retinal potentials in response to full-field flashes have been used in the clinical assessment of retinal function for some decades (Marmor et al., 1989; Marmor et al., 2009) and are therefore well described. These potentials, which are seen in the electroretinogram (ERG), reflect the summed activity of the retinal network and arise from different processing stages (Frishman, 2013). The first negative deflection of the human ERG in response to a light flash is the a-wave, which originates from the photoreceptors (Perlman, 2001; Frishman, 2013). It is truncated by the rising flank of the positive b-wave, a potential that is mostly driven by ON bipolar cells (Sieving et al., 1994; Frishman, 2013; Vukmanic et al., 2014). At the offset of long duration light flashes, a potential referred to as the d-wave can be seen: this positive deflection has its origin in the OFF bipolar cells (Sieving et al., 1994; Perlman, 2001; Frishman, 2013); in the photopic ERG, a contribution of the cone receptors is assumed (Evers and Gouras, 1986; Frishman, 2013).

A peculiarity of the ERG is the oscillatory potential, an onset locked high frequency oscillation that has first been described by Fröhlich (1914). It is characterized as a millisecond precise oscillation on the rising flank of the b-wave, with a frequency centered 
around $120 \mathrm{~Hz}$, and sometimes described as involving frequencies up to $200 \mathrm{~Hz}$ (Kozak, 1971; Munk and Neuenschwander, 2000; Todorov et al., 2016). Mechanisms and cellular origin of the oscillatory potential are still unknown, an involvement of ganglion, amacrine and bipolar cells, possibly in a negative feedback loop, is discussed (Doty and Kimura, 1963; Perlman, 2001; Kenyon et al., 2003; Frishman, 2013). Kozak (1971) describes a similar but slower oscillation $(75-125 \mathrm{~Hz})$ in response to light offset.

Retinocortical propagation The visual system involves several stages of processing: commencing in the retina, information is passed to the LGN in thalamus and then projected to the occipital cortex. There is evidence, that the retinal oscillatory potential is directly transmitted to visual cortex (Lopez and Sannita, 1997; Castelo-Branco et al., 1998; Sannita et al., 1999; Heinrich and Bach, 2001; Neuenschwander et al., 2002; Todorov et al., 2016). Other studies, however, come to the conclusion that retinal and cortical gamma are two distinct processes (Doty and Kimura, 1963; Molotchnikoff et al., 1975; Heinrich and Bach, 2004). Reconciling these opposite findings, it has been proposed that two different types of cortical high frequency activity exist, one inherited from the retina and one generated locally after visual stimulation. Munk and Neuenschwander (2000) suggest that the oscillatory potential, incorporating frequencies from 60 to $120 \mathrm{~Hz}$, is transmitted from the retina to cortex, whereas slower cortical gamma activity below $60 \mathrm{~Hz}$ is generated locally. A recent study by Saleem et al. (2017) reports that cortical narrowband gamma responses are inherited from thalamus and proposes different channels for thalamocortical (narrowband) and corticocortical (broadband) information transfer.

The present study investigates the retinal and cortical responses to light flash onsets and offsets and aims to compare their temporal dynamics and oscillatory patterns. The simultaneous recording of retinal and cortical activity further enables a direct comparison of retinal and cortical high frequency activity and its propagation through the visual system. 


\section{Methods}

Participants 10 healthy participants (four female, average age 34.1 years; s.d. = 6.31) took part in the study. 6 participants were contact lens wearers, since experience showed that they usually tolerate the eye electrode used to record the ERG very well. Contact lens wearers did not wear their lenses during the experiment. All participants provided written informed consent and the study was approved by the Ethical Committee of Central Denmark Region and carried out in accordance with the Declaration of Helsinki.

Experimental design and data acquisition The experimental stimuli were full field light flashes which were presented using the Presentation software (Neurobehavioral Systems, Inc., Berkeley, CA). The white flashes had a duration of $480 \mathrm{~ms}$ and were followed by a black screen which was shown for a random time interval between 2000 and $2500 \mathrm{~ms}$. A total of 250 flashes was shown and the experiment lasted approximately $12 \mathrm{~min}$. The flashes were projected onto a screen inside the MEG chamber using a ProPixx projector (VPixx Technologies Inc., Saint-Bruno, Canada) with a $60 \mathrm{~Hz}$ refresh rate and symmetric rise and fall times. Participants were seated in an upright position, the projection screen was at $70 \mathrm{~cm}$ distance from the subjects. The flashes were as full-field as possible subtending the central $28.01^{\circ}$ (vertical extent) and $47.77^{\circ}$ (horizontal extent) of the visual field and had a brightness of $280 \mathrm{~cd} \mathrm{~m}^{-2}$.

MEG data was recorded using a 306-channel MEG system (102 magnetometers and $2 \times 102$ gradiometers, Elekta Neuromag TRIUX, Elekta Instruments, Stockholm, Sweden) in a magnetically shielded room. Data was sampled at $5 \mathrm{kHz}$ with a recording bandwidth of 0.1-1650 Hz. Bilateral ERG was recorded using disposable Dawson-TrickLitzkow (DTL) fiber electrodes. Additionally, horizontal and vertical electrooculogram (HEOG and VEOG) were recorded using a bipolar montage. The ERG electrodes were referenced to the ipsilateral HEOG. Prior to data acquisition, the head position indicator (HPI) coils and three fiducial points (left and right periauricular points and nasion) were digitized using a Polhemus Fastrak 3D scanner (Polhemus, Colchester, VT, USA) for later coregistration with the strucutral magnetic resonance image (MRI) of the 
subjects. The on- and offsets of the flashes were recorded with a photodiode during the whole experiment.

Data analysis Analysis of MEG and ERG data was conducted using the opensource toolboxes FieldTrip (Oostenveld et al., 2010) and NUTMEG (Dalal et al., 2004; Dalal et al., 2011) for MATLAB. Epochs of light onsets and offsets were identified using the photodiode traces. Trials with eye-movements were rejected based on the HEOG and VEOG activity. Subsequently, trials including muscle artifacts or MEG channel jumps were excluded as well, leaving on average 183.4 trials $(s t d=24.06)$ per subject and condition. The data was downsampled to $1000 \mathrm{~Hz}$.

For ERG data analysis, only data from the left ERG was used. Data was baseline corrected and detrended and the epochs were then averaged with respect to light onset and offset. The peak latencies for the retinal potentials (a-, b- and d-wave) were identified on the averaged time series for every subject. A paired samples Wilcoxon signed rank test was conducted on the b-wave and d-wave measurements, as well as on the a-wave and d-wave peaks. To obtain the oscillatory potentials after light onset, ERG data was highpass-filtered at $55 \mathrm{~Hz}$ (Hanning windowed FIR filter, onepass-zerophase, $6 \mathrm{~Hz}$ transition width). For light offset, data was highpass-filtered at $75 \mathrm{~Hz}$ and lowpass-filtered at $95 \mathrm{~Hz}$ using the same filter definitions.

For MEG data analysis, only the 102 magnetometers were used. Boundary element head models with three layers (brain, skull, scalp) were constructed for every subject based on the individual structural MRI using OpenMEEG (Gramfort et al., 2010; Gramfort et al., 2011). The source grid spanning the whole brain had a resolution of $10 \mathrm{~mm}$. Sources were reconstructed using the linearly constrained minimum variance (LCMV) beamformer (Van Veen et al., 1997) with normalized weights (Van Veen et al., 1997; Sekihara and Nagarajan, 2008). The covariance matrices passed to the beamformer were computed based on the Minimum Covariance Determinant estimator, providing a robust covariance matrix estimate. The beamforming approach was combined with the Hilbert transform to acquire source space Hilbert amplitude and phase for five frequency bands: 55-75, 75-95,105-125,125-145, and 155-195 Hz. To gener- 
ate these frequency bands, separate high- and lowpass filters were adopted (Hanning windowed FIR filter, onepass-zerophase, $6 \mathrm{~Hz}$ transition width). For every frequency band and condition, a spatial filter was constructed as described above, and the single trials were projected through the filter to yield virtual electrodes at every grid point. Subsequently, the time courses of the virtual electrodes were Hilbert transformed, providing amplitude estimates for every frequency band. Intertrial coherence (ITC) was computed based on the Hilbert phase estimates. To allow for comparison of retinal and cortical high frequency activity, the ERG data was filtered and Hilbert transformed in the same manner.

For both MEG and ERG data, the Hilbert amplitude time courses were normalized against the distribution of baseline time points using the Wilcoxon rank sum test for every frequency band and subject. The z-values obtained from this step were tested against the baseline distribution across subjects with the Wilcoxon rank sum test. The derived statistics were corrected for multiple comparisons by controlling the false discovery rate (FDR). ITC peaks were identified for every subject; in source space, the maximal ITC was searched among all occipital voxels based on the Automated Anatomical Labeling (AAL) atlas (Tzourio-Mazoyer et al., 2002) for every subject. Due to a polyphasic response, the retinal light offset ITC for one participant was smoothed by lowpass filtering the ITC time series (cutoff: $40 \mathrm{~Hz}$ ) to identify a clear peak latency. Light onset and offset ITC peak times were then tested using the paired sample Wilcoxon signed rank test for both retinal and cortical activity.

\section{Results}

Retinal evoked potentials The across-subjects average of retinal activity following light onset revealed the characteristic ERG flash response (Figure 1A). The negative a-wave had an average latency of $24.2 \mathrm{~ms}$ across subjects, followed by the positive b-wave at $79.9 \mathrm{~ms}$. The data also shows the c-wave, which is generally seen with longer flashes, but of no further interest in this study. Figure 1B shows the retinal response to light offset with the d-wave peaking at $25.2 \mathrm{~ms}(n=8$ : for two subjects 
the peak was not identifiable). The comparison of d-wave and b-wave latencies using a non-parametric paired-sample test (Wilcoxon signed-rank test) revealed a significant difference $(R=36, p=0.0078)$ with the d-wave being faster across subjects. There was no peak latency difference between the a-wave and the d-wave: $R=13, p=0.5469$.

Retinal and cortical high frequency activity As illustrated in Figure 2A, highpass filtering the light onset ERG data at $55 \mathrm{~Hz}$ reveals a high frequency burst. This oscillatory potential has high inter-trial and even inter-individual fidelity and can thus still be seen in the average across subjects. A comparable pattern in the light-off data is only evident when looking at a narrow frequency band of $75-95 \mathrm{~Hz}$ (Figure $2 \mathrm{~B}$ ). This is supported by the fact that across subjects, only this frequency band shows a significant increase in ITC after light offset compared to baseline by adopting a Wilcoxon rank sum test (Figure 2C).

To evaluate high frequency activity in the MEG data, Hilbert amplitudes of five frequency bands were computed in source space. In response to light onset, all but the highest frequency band show significant increases in Hilbert amplitude (in comparison to baseline, Wilcoxon rank sum test across subjects). Figure 3A shows this broadband response (the black boxes indicate significant time periods, $p<0.001$, FDR-corrected) and suggests that the activity in higher frequency bands occurs earlier than changes in lower frequencies. This gamma band activity spans occipital regions, including V1 as well as visual upstream regions (Figure 3B, masked for $p<0.001$, FDR-corrected). The brain's activity following light offset comprised a narrowband response of $75-95 \mathrm{~Hz}$ localized to occipital cortex (Figure 3C and D, masked for $p<0.001$, FDR-corrected), which is the same frequency band as for the retinal response to light offset.

It has recently been shown in rats that the flash-induced retinal oscillatory potential is transferred via the optic nerve to the occipital cortex (Todorov et al., 2016). To investigate and compare retinal and cortical oscillatory potentials in this study, we calculated intertrial coherence for those frequency bands that revealed significant power increases (see above). Figure 4A shows the ITC time course in response to light onset 

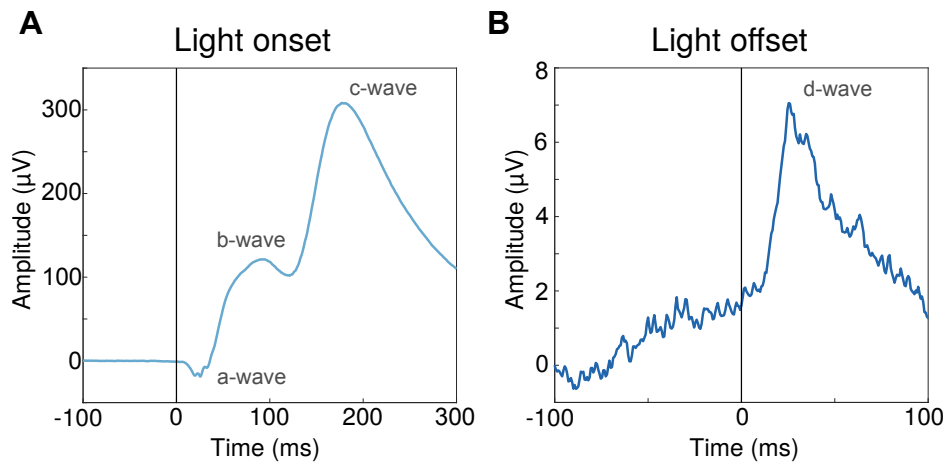

Figure 1: Retinal evoked potentials. A Retinal potentials following flash onset, averaged across subjects. B ERG response to light offset, averaged across subjects.

A

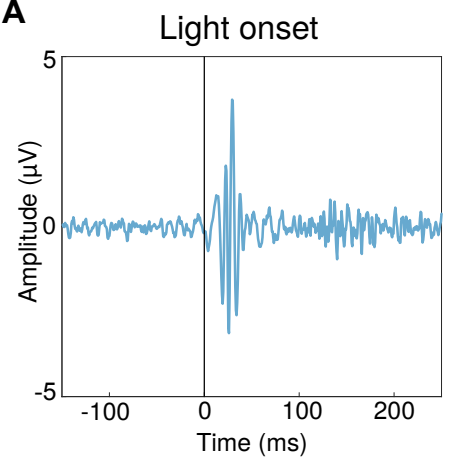

B

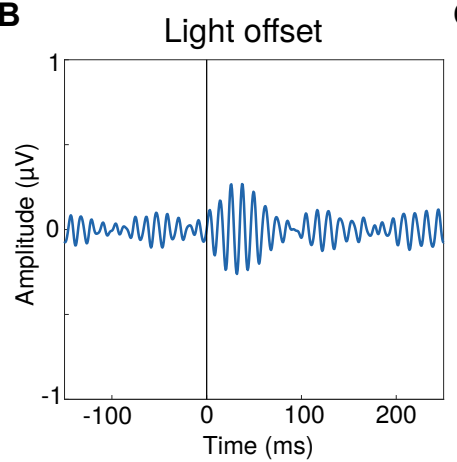

C

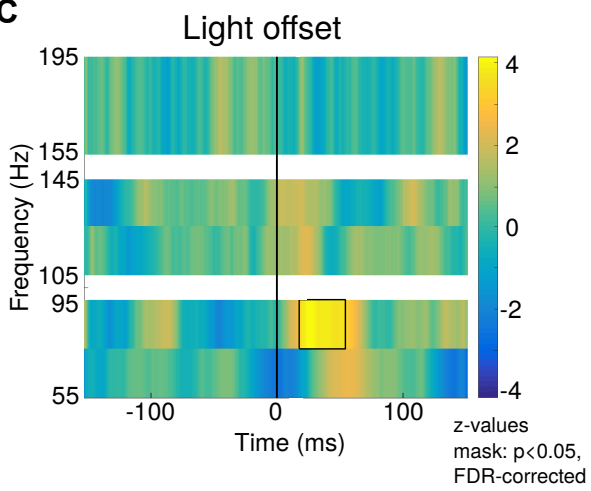

Figure 2: Retinal oscillatory potentials. A Oscillatory potential after light onset. The data is highpass filtered at $55 \mathrm{~Hz}$ and averaged across subjects. B The oscillatory potential after light offset. Data is bandpass filtered from 75 to $95 \mathrm{~Hz}$ and averaged across subjects. C Intertrial coherence for light offset, tested against baseline across subjects (Wilcoxon rank sum test), black box marks significant area $(p<0.05$, FDR-corrected). 
bioRxiv preprint doi: https://doi org/10.1101/153551; this version posted June 21, 2017. The copyright holder for this preprint (which was not certified by peer review) is the author/funder, who has granted bioRxiv a license to display the preprint in perpetuity. It is made available under aCC-BY 4.0 International license.

A

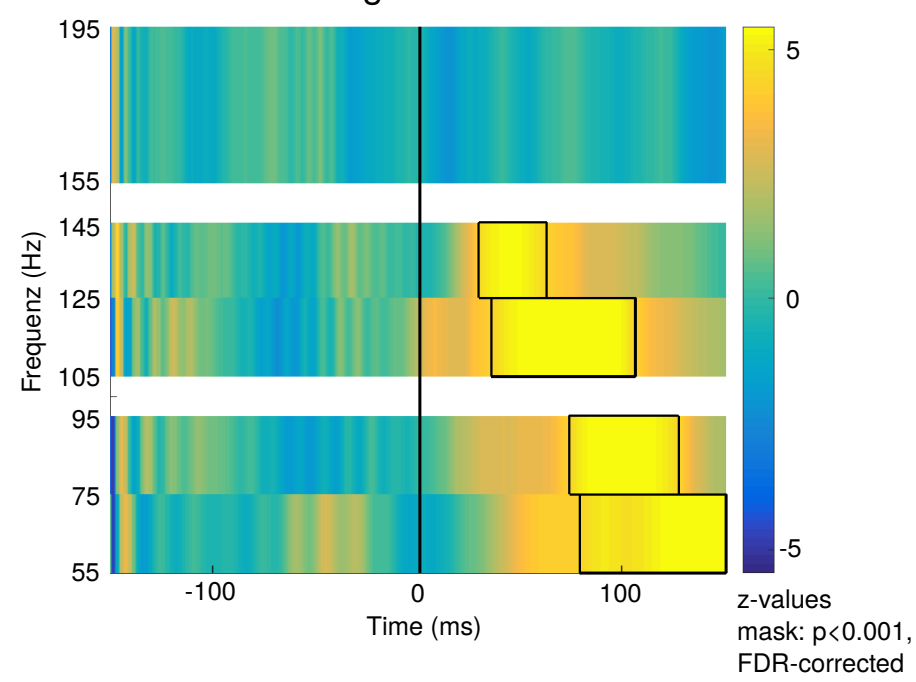

B
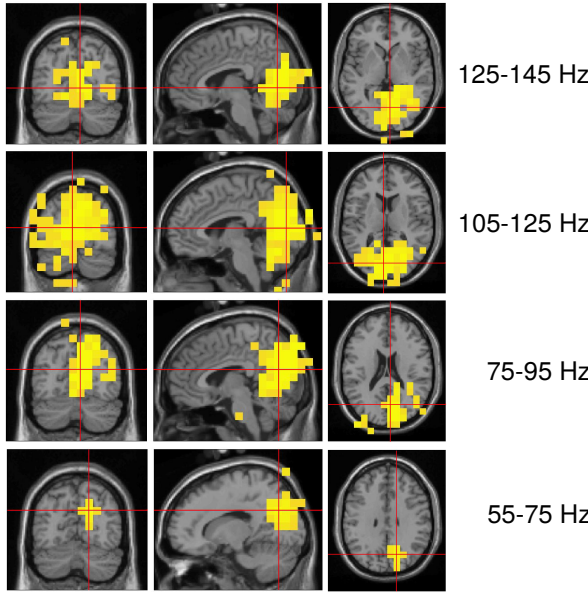

$105-125 \mathrm{~Hz}$

$75-95 \mathrm{~Hz}$

$55-75 \mathrm{~Hz}$
C

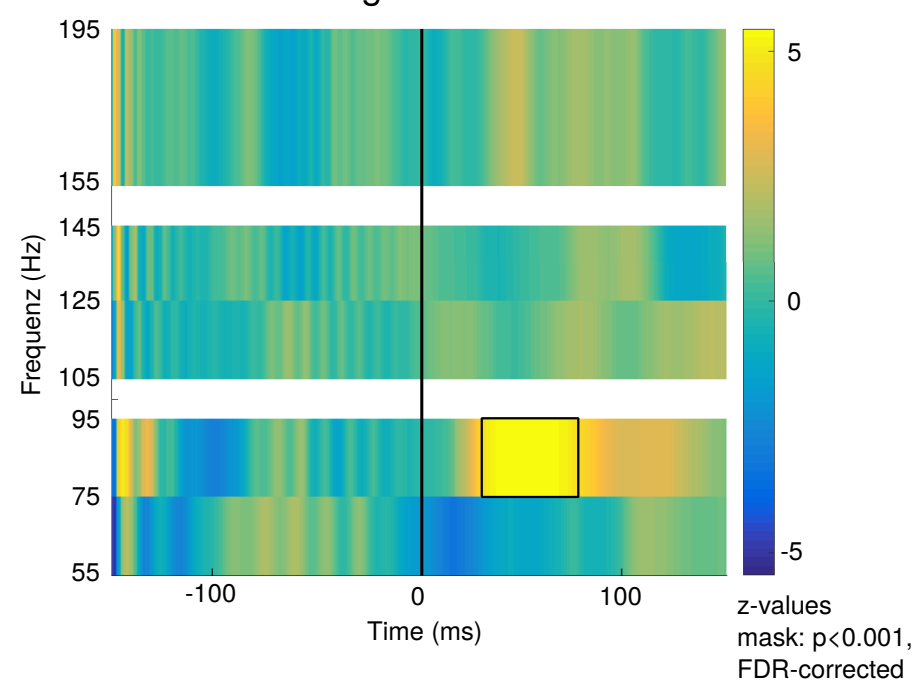

D

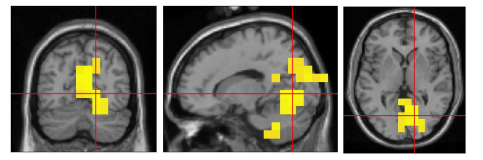

$75-95 \mathrm{~Hz}$

Figure 3: Occipital high frequency activity. Both light onset and light offset evoked cortical high frequency activity. Hilbert amplitudes in different frequency bands are tested against baseline across subjects with the Wilcoxon rank sum test. Depicted for each frequency band is the time courses of the maximum voxel. A Occipital high frequency activity following light onset. Black boxes indicate time periods with $p<0.001$ (FDR-corrected). B Voxels with significant activity in the different frequency bands following light onset, activity is masked for $p<0.001$. The red cross hairs mark the maximum voxels. $\mathbf{C}$ High frequency activity following light offset in visual cortex. D Localization of flash offset activity, the cross hairs mark the maximum voxel. 
for the ERG (depicted in pale blue, median and inter-quartile range across subjects) and for occipital cortex (pale red), where the cortical ITC time course is computed as the across-subjects median of individual occipital maximum ITC. It is evident that the retinal oscillatory potential after light onset is followed by phase consistent activity in the cortex. This pattern is most obvious for the higher frequency bands: in the 105$125 \mathrm{~Hz}$ band, the cortical ITC peak follows after $51.0 \mathrm{~ms}$ (median difference between retinal and cortical peak across subjects, number of peak relations $n=9$, since the retinal onset ITC peak was not identifiable for one subject). In the $125-145 \mathrm{~Hz}$ band, cortical activity follows the retinal ITC peak after $32.0 \mathrm{~ms}(n=9)$. As illustrated in Figure 4B, light offset led to a comparable pattern: an increase in trial-wise phase consistency in the ERG (depicted in dark blue) is followed by increased ITC in occipital cortex $21.0 \mathrm{~ms}(n=7)$, which is shown in dark red (also compare Table 1 ).

A
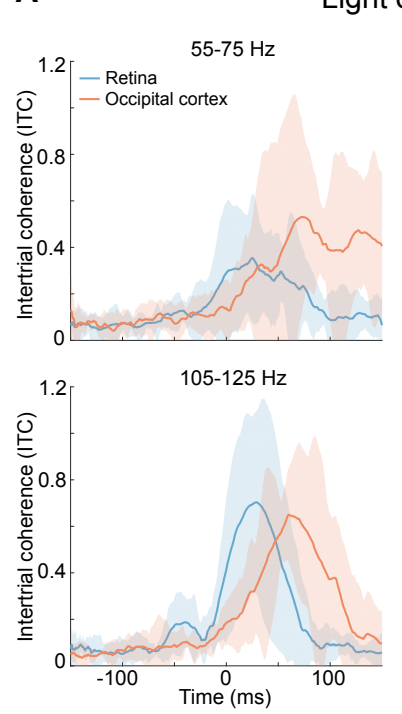

Light onset

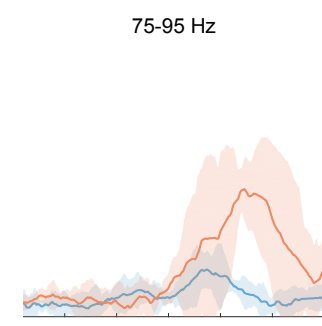

$125-145 \mathrm{~Hz}$

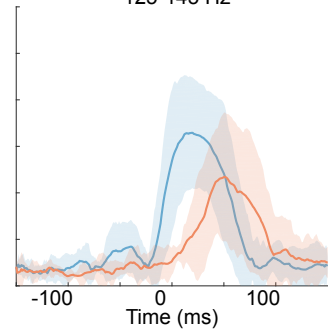

B Light offset

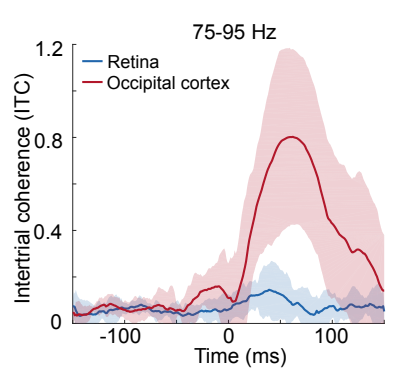

Figure 4: Retinal and cortical intertrial coherence. Shown are median time courses across subjects, shaded areas represent the inter-quartile range. A Time courses of ITC following light onset for the frequency bands with significant activity (cf. Figure 3A). Retinal ITC courses are depicted in pale blue, the cortical ITC in pale red. Cortical ITC time courses represent the median across subjects' individual maximum ITC activity in occipital cortex. B ITC time course in response to light offset for the $75-95 \mathrm{~Hz}$ narrowband response (cf. Figure $2 \mathrm{C}$ and $3 \mathrm{C}$ ). The retinal response is shown in dark blue, the median response across individual occipital maximum voxels is shown in dark red.

High frequency activity: comparing light onset and offset To assess whether the latencies of retinal and cortical high frequency bursts differ between light onset and offset, ITC peak times were tested across subjects with the Wilcoxon signed rank 
bioRxiv preprint doi: https://doi.org/10.1101/153551; this version posted June 21, 2017. The copyright holder for this preprint (which was not certified by peer review) is the author/funder, who has granted bioRxiv a license to display the preprint in perpetuity. It is made available under aCC-BY 4.0 International license.

test. Since light offset was characterized by a narrowband response $(75-95 \mathrm{~Hz})$, the different onset frequency bands were all tested against this one offset frequency band. The retinal ITC peak latencies (cf. Figures 5A) show no significant difference between light onset and offset for the frequency bands of $55-75 \mathrm{~Hz}, 75-95 \mathrm{~Hz}$, and $125-145 \mathrm{~Hz}$ (cf. Table 1). In the $105-125 \mathrm{~Hz}$ frequency band, the light onset ITC peak $(27.0 \mathrm{~ms}$, $n=9)$ was significantly earlier than the $75-95 \mathrm{~Hz}$ light offset peak $(34.0 \mathrm{~ms}, n=7$; $R=0.0, p=0.0312)$. In the cortex, however, the offset response at $75-95 \mathrm{~Hz}$ shows a significantly earlier ITC peak time $(57.0 \mathrm{~ms}, n=10)$ than the light onset oscillatory potentials of the $75-125 \mathrm{~Hz}$ frequency range $(75-95 \mathrm{~Hz}: 71.5 \mathrm{~ms}, n=10): R=49$, $p=0.0254$ and $105-125 \mathrm{~Hz}: 77.0 \mathrm{~ms}(n=10), R=40, p=0.0352$. There was no difference for the other light onset frequency bands of $55-75 \mathrm{~Hz}$ and $125-145 \mathrm{~Hz}$ (cf. Table 1 and Figure 5B). Thus, whereas offset high frequency oscillations peak faster than onset responses in the brain, they seem to peak equally fast or even slower in the retina. This pattern suggests that the narrowband light offset response is transferred faster to cortex than the onset response: the light offset ITC peaks significantly later in the retina than the light onset response for $105-125 \mathrm{~Hz}$, but earlier in the brain.

A
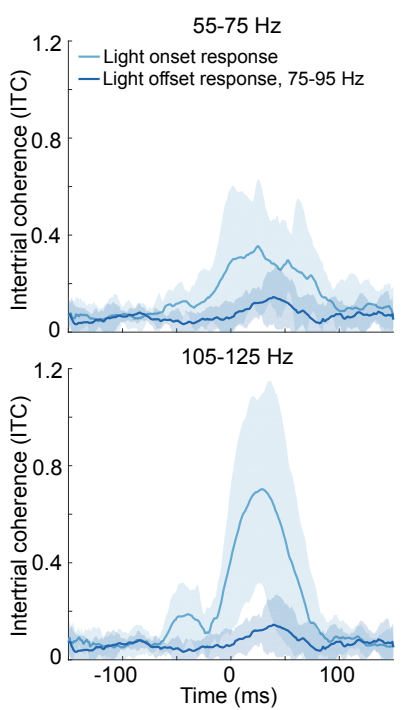

Retina

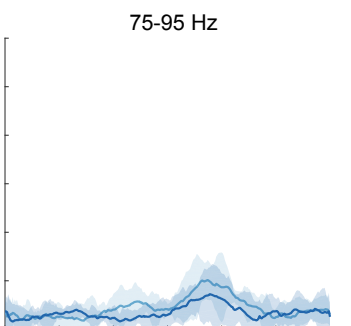

$125-145 \mathrm{~Hz}$

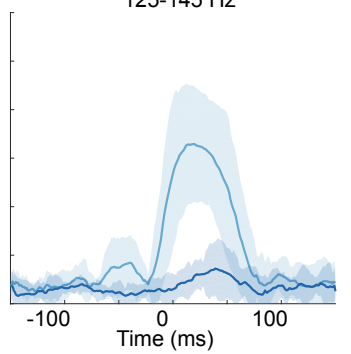

B

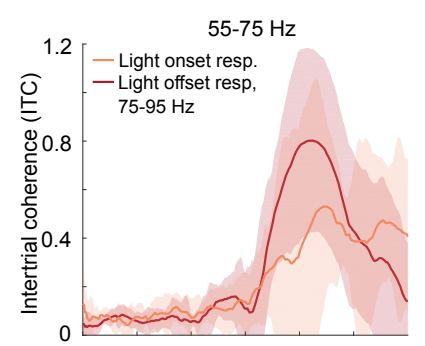

$105-125 \mathrm{~Hz}$

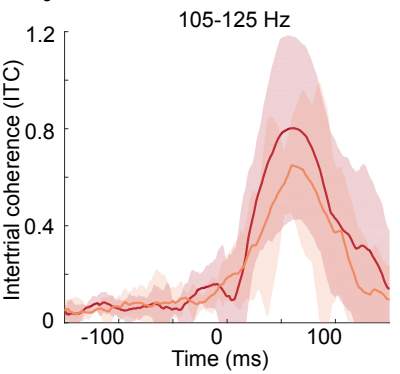

Occipital cortex

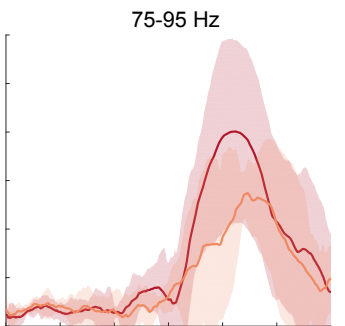

$125-145 \mathrm{~Hz}$

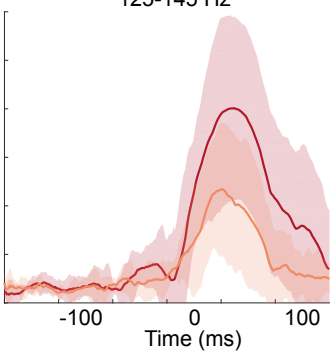

Figure 5: Comparison of intertrial coherence for light onset and offset. Shown are median time courses across subjects, shaded areas represent the inter-quartile range. A Retinal responses to flash onset (pale blue) and offset (dark blue). The light onset responses are shown for the frequency bands with significant activity (cf. Figure 3A). The light offset response is shown for $75-95 \mathrm{~Hz}$ in all four panels, as follows from Figure 2C. B Occipital ITC responses for light onset (pale red) and light offset (dark red). Presentation as in A. 
Table 1: Intertrial coherence peak latencies. Retinal and cortical peak times for the ITC of different frequency bands for both light onset and offset. Shown are peak times for frequency bands with significant activity (cf. Figures 2 and 3). Specified as well are the number of identifiable peak times per condition (subjects, $n$ ). The last column shows the results from a Wilcoxon signed rank test of light onset peak latencies of the different frequency bands against the 75-95 Hz light offset peak latency. The last section of the table shows the propagation times (median across subjects), defined as the difference between the cortical and retinal ITC peak time.

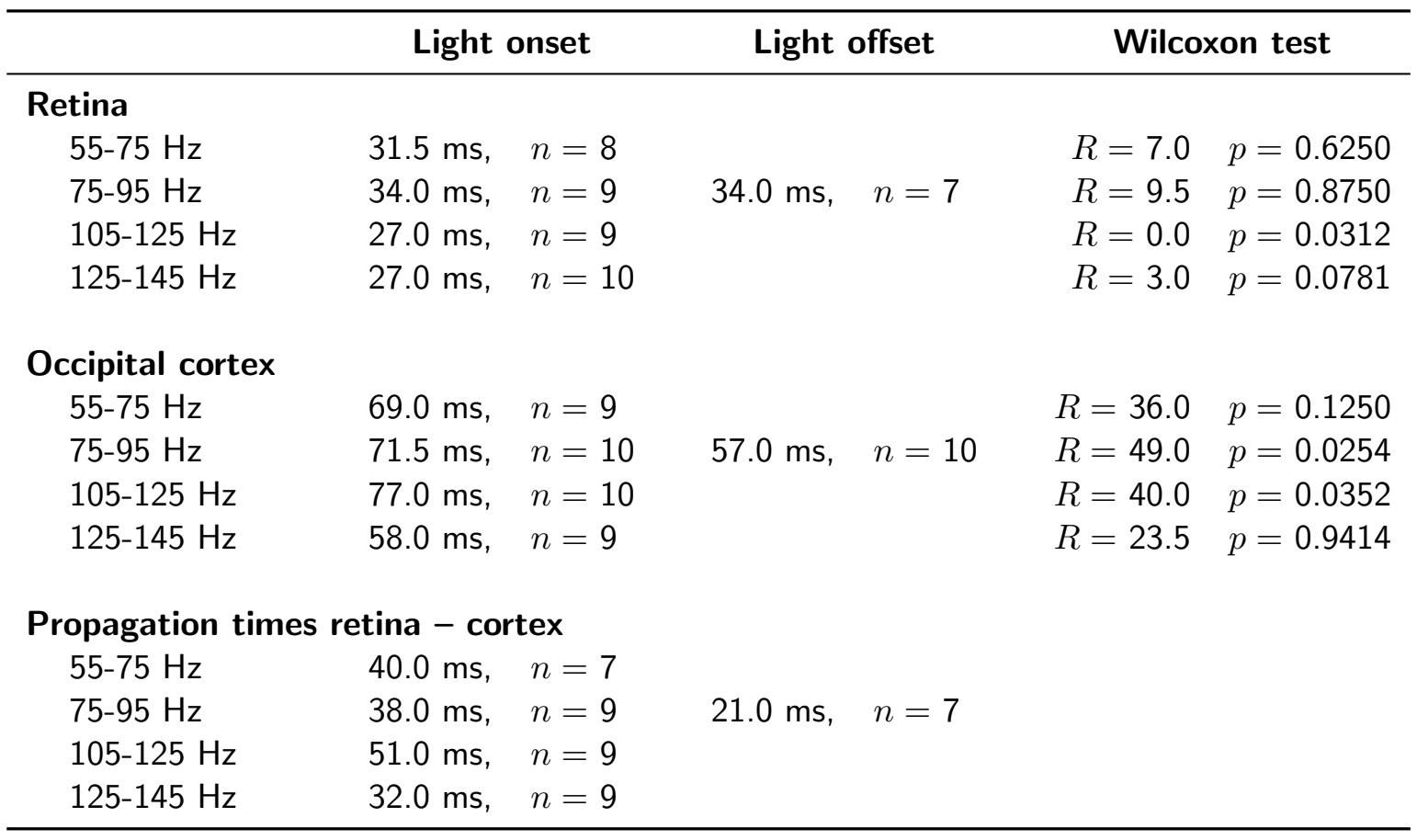




\section{Discussion}

There are studies showing advantages for the processing of darks on different levels of the visual system (e.g., Zemon et al., 1988; Yeh et al., 2009; Jin et al., 2011; Komban et al., 2011; Nichols et al., 2013; Komban et al., 2014), however, evidence from the human brain is still sparse. In the present study, we simultaneously recorded retinal and cortical activity to flash onsets and offsets in order to investigate the differences and similarities in the respective activity patterns. The focus was hereby on oscillatory activity in the high frequency range in order to map the retinal oscillatory potential (Fröhlich, 1914) and its potential transmission to occipital cortex (Todorov et al., 2016).

Are darks processed faster than lights? Especially behavioral studies suggest a faster processing of darks compared to lights: reactions to dark objects and light decrements are faster and more accurate (Blackwell, 1946; Chubb and Nam, 2000; Buchner and Baumgartner, 2007). On cortical, thalamic, and retinal level, evidence for faster processing of darks is mixed (e.g., Lankheet et al., 1998; Chichilnisky and Kalmar, 2002; Gollisch and Meister, 2008; Jin et al., 2008; Yeh et al., 2009), although numerous studies report greater neural resources for the processing of darks (e.g., Balasubramanian and Sterling, 2009).

We compared retinal and cortical ITC peak times in the high frequency range as well as retinal evoked potentials in response to light onsets and offsets to conclude on temporal differences in processing and propagation in the visual system.

The first obvious difference between light onset and offset high frequency activity is the frequency range itself: whereas the retinal oscillatory potential in response to light onset contains frequencies from 55 to $195 \mathrm{~Hz}$, the retinal high frequency activity following light offset is restricted to the $75-95 \mathrm{~Hz}$ band. A very similar pattern emerges in visual cortex: the light onset high frequency response comprises frequencies between 55 and $145 \mathrm{~Hz}$, while the flash offset activity is limited as well to $75-95 \mathrm{~Hz}$. The cortical onset activity's upper limit at $145 \mathrm{~Hz}$ is presumably due to a lower signal-to-noise ratio related to very high frequency content in MEG recordings.

In visual cortex, the narrowband $75-95 \mathrm{~Hz}$ light offset response is faster than the ac- 
tivity in the two main frequency bands for light onset, $75-95 \mathrm{~Hz}$ and $105-125 \mathrm{~Hz}$. For the $55-75 \mathrm{~Hz}$ and $125-145 \mathrm{~Hz}$ flash onset response, no significant temporal difference to light offset was found. This finding corroborates the assumption that darks are processed faster than lights at the cortical level (Komban et al., 2014).

In the retina, the picture is not as homogeneous: while there is no significant difference concerning light onset and offset peak times in most frequency bands, the main onset frequency band of $105-125 \mathrm{~Hz}$ has an earlier peak time than the $75-95 \mathrm{~Hz}$ offset response. Thus, the retinal high frequency activity shows an opposite pattern to the cortical oscillatory responses.

When looking at the retinal evoked potentials, studies on the generators of these potentials suggest to compare the b-wave (light onset) to the d-wave (light offset), since both are presumably driven by bipolar cells (Sieving et al., 1994; Perlman, 2001; Frishman, 2013; Vukmanic et al., 2014). In our data, the d-wave is significantly faster than the b-wave. However, the fact that the b-wave peaks as late as $79.9 \mathrm{~ms}$ which is even later than the ITC peaks of high frequency activity in cortex (58.0 to $77.0 \mathrm{~ms}$ ) raises the question whether this is a just comparison. The d-wave latency $(25.2 \mathrm{~ms})$ is around $10 \mathrm{~ms}$ earlier than the latency of the offset oscillatory potential $(34.0 \mathrm{~ms})$. The same is true for the light onset activity when comparing the a-wave latency $(24.2 \mathrm{~ms})$ to the peak times of the different frequency bands (27.0$31.5 \mathrm{~ms}$ ), whereas the b-wave peaks over $45 \mathrm{~ms}$ after this high frequency activity. When taking the oscillatory potentials as an anchor, considering that they are reflecting a rather late mechanism in retinal processing (possibly feedback loops between different retinal cell types, see Doty and Kimura, 1963; Perlman, 2001; Kenyon et al., 2003; Frishman, 2013), then it becomes apparent, that the d-wave and b-wave might not reflect related cell activities after all. When instead comparing the d-wave to the a-wave, there is no difference in timing, however, the a-wave and d-wave are supposedly not generated by the same cell population (Sieving et al., 1994; Perlman, 2001; Frishman, 2013).

Looking at the peak latencies of oscillatory activity, our data suggests faster processing of darks on the cortical, but not on the retinal level. The evidence on retinal level is 
mixed: while the main onset frequency band peaks faster than high frequency activity in response to light offset, a comprehensive interpretation of retinal potential latencies is questionable.

Emerging from the retinal and cortical peak latencies of oscillatory activity, the propagation time of information is faster for darks $(21.0 \mathrm{~ms})$ than lights $(32.0$ to $51.0 \mathrm{~ms})$. The light onset propagation times replicate previous findings of Heinrich and Bach (2001), who reported a time lag of $48 \mathrm{~ms}$. The faster propagation of light offset information suggests a thalamic role, which is supported by the finding of a faster processing for light decrements in the LGN of cats (Jin et al., 2011). Correspondingly, Xing et al. (2010) described a temporal advantage for darks in the thalamic input layer of V1 in macaque monkeys, however, it must be noted that they report no time differences in upstream visual areas. One possible explanation for the faster transmission time for darks could be a lesser informational content for darks compared to lights. Light onset could evoke more features of visual scene processing, e.g., stereo vision, which renders potential thalamic processing faster. Alternatively, the faster transmission times could be explained by asymmetries in the $\mathrm{ON}$ and $\mathrm{OFF}$ pathways as it has been suggested that more neural resources are allocated to the OFF pathway (Balasubramanian and Sterling, 2009).

In summary, our results strengthen findings of faster processing for darks in visual cortex and thereby deliver a possible explanation for any behavioral advantages of darks over lights. On the retinal level, we did not find faster processing of light decrements. Instead, light increments seem to be processed equally fast or even faster than light decrements. Due to the fact that the ERG represents the summed activity of different cell types and due to the lack of knowledge regarding the origin of the retinal oscillatory potential, it is hardly possible to speculate about the precise underlying retinal mechanisms of this finding. Information transmission to visual cortex, however, seems to happen faster for light offset than onset, suggesting a thalamic involvement.

Are retinal oscillatory potentials transmitted to cortex? Whether the retinal oscillatory potential gets transmitted to visual cortex has been a controversial topic. 
Some studies come to the conclusion that this is not the case: Heinrich and Bach (2004), for example, describe different peak frequencies in retina and cortex and Molotchnikoff et al. (1975) report a lack of cortical high frequency activity following flash-stimulation despite a clear retinal response. Doty and Kimura (1963) find a link between retinal and cortical gamma band activity in monkeys but not in cats. Other studies, however, show strong evidence for a propagation of the oscillatory potential through the visual system (Lopez and Sannita, 1997; Sannita et al., 1999; Heinrich and Bach, 2001; Munk and Neuenschwander, 2000; Neuenschwander et al., 2002; Koepsell et al., 2009). Castelo-Branco et al. (1998) described strong correlations between retinal, thalamic and cortical high frequency activity $(60-120 \mathrm{~Hz})$ in cats. More recently, Todorov et al. (2016) reported high coherence between the retina, the optic chiasm and visual cortex in rats, however, they note different wave shapes in these three stages of the visual system and therefore argue against a merely passive spread of the oscillatory potential. The current data shows evoked oscillatory activity following light onset and offset in both retina and cortex. This activation comprises similar frequency bands in the retina and in visual cortex: the light onset response is broadband in the retina $(55-195 \mathrm{~Hz})$ as well as in cortex $55-145 \mathrm{~Hz}$. The lack of significant flash-evoked activity in the 155-195 Hz frequency band in visual cortex is presumably due to the low signal-tonoise ratio of such high frequencies. Equivalently, the offset response is restricted to the same frequency band $(75-97 \mathrm{~Hz})$ in both retina and visual cortex. This activation pattern is consistent with a propagation of the retinal oscillatory potential to visual cortex. Furthermore, the faster propagation time for light offset responses suggests thalamic involvement and indicates that the propagation of the oscillatory potential to the visual cortex is not a mere passive spread (Todorov et al., 2016).

\section{The role of narrowband and broadband gamma responses in the visual sys-}

tem As outlined above, light onset evoked a broadband high frequency response in the retina and visual cortex, whereas light offset was followed by a narrowband response in the $75-95 \mathrm{~Hz}$ range. Narrowband oscillatory activity in the visual system has been observed in response to stationary or moving grating stimuli (e.g., Adjamian et al., 2004; 
Hoogenboom et al., 2006; Muthukumaraswamy et al., 2010) and has been shown to vary in peak frequency depending on different stimulus features like eccentricity or movement (Swettenham et al., 2009; van Pelt and Fries, 2013). Narrowband gamma responses were also described with focused attention (Vidal et al., 2006). However, there is a debate about the origin as well as functional implication of such narrowband responses, for example, about the question if they are solely induced by grating stimuli (Hermes et al., 2014; Hermes et al., 2015), or also by natural stimuli (Brunet et al., 2014). In the present study, the narrowband gamma response was elicited by light offset and is thus presumably linked to the OFF pathway of the visual system. This finding suggests that gratings could trigger an exceeding involvement of the OFF pathway which is apparent in cortical narrobwand responses.

A recent paper shows narrowband gamma oscillations in the visual system of mice: Saleem et al. (2017) report that visual broadband and narrowband activity is not correlated: with higher contrast, broadband gamma increases while narrowband oscillations decrease. They show that the narrowband gamma response is inherited from thalamus and propose a model with two different channels for information transfer: the narrowband gamma enabling thalamocortical communication and the broadband gamma allowing for corticocortical interactions. In the present study, we show that both narrowband and broadband gamma are transmitted from retina to cortex. However, they still comprise different information, possibly even different levels of informational value. Light contains per se more information than darkness, and supposedly evokes more features in visual scene processing, which could be an explanation why more frequency bands are involved in carrying the information, as well as why the transmission is faster for light offset responses.

In summary, our data supports faster processing of darks on the cortical but not retinal level. The oscillatory potential gets transmitted to visual cortex, and this propagation is faster for light offset responses. Furthermore, we show that light onset high frequency activity comprises a broad range of frequencies, whereas the response to light offset evokes a narrowband oscillation in the range of $75-95 \mathrm{~Hz}$ in both retina 
and cortex.

Acknowledgements We thank Christopher Bailey for his assistance in data collection and Tzvetan Popov and Ursula Lommen for their help with a pilot recording. Further, we thank Juan Vidal for valuable discussions about this study. This work was supported by the Zukunftskolleg of the University of Konstanz, ERA-Net NEURON via the Bundesministerium für Bildung und Forschung (BMBF grant 01EW1307), and the European Research Council (Starting Grant 640488).

\section{References}

Adjamian P, Holliday IE, Barnes GR, Hillebrand A, Hadjipapas A, Singh KD (2004) Induced visual illusions and gamma oscillations in human primary visual cortex. European Journal of Neuroscience 20:587-592.

Ahmad KM, Klug K, Herr S, Sterling P, Schein S (2003) Cell density ratios in a foveal patch in macaque retina. Visual Neuroscience 20:189-209.

Balasubramanian V, Sterling P (2009) Receptive fields and functional architecture in the retina. The Journal of Physiology 587:2753-2767.

Benardete EA, Kaplan E (1997) The receptive field of the primate P retinal ganglion cell, I: Linear dynamics. Visual Neuroscience 14:169-185.

Benardete EA, Kaplan E (1999) The dynamics of primate M retinal ganglion cells. Visual Neuroscience 16:355-368.

Blackwell HR (1946) Contrast thresholds of the human eye. JOSA 36:624-643.

Borghuis BG, Ratliff CP, Smith RG, Sterling P, Balasubramanian V (2008) Design of a neuronal array. Journal of Neuroscience 28:3178-3189.

Bowen RW, Pokorny J, Smith VC (1989) Sawtooth contrast sensitivity: Decrements have the edge. Vision Research 29:1501-1509.

Brunet N, Vinck M, Bosman CA, Singer W, Fries P (2014) Gamma or no gamma, that is the question. Trends in Cognitive Sciences 18:507-509.

Buchner A, Baumgartner N (2007) Text-background polarity affects performance irrespective of ambient illumination and colour contrast. Ergonomics 50:1036-1063.

Burkhardt DA, Fahey PK, Sikora MA (2007) Retinal bipolar cells: temporal filtering of signals from cone photoreceptors. Visual Neuroscience 24:765-774.

Castelo-Branco M, Neuenschwander S, Singer W (1998) Synchronization of visual responses between the cortex, lateral geniculate nucleus, and retina in the anesthetized cat. Journal of Neuroscience 18:6395-6410.

Chichilnisky EJ, Kalmar RS (2002) Functional asymmetries in ON and OFF ganglion cells of primate retina. The Journal of Neuroscience 22:2737-2747. 
Chubb C, Nam JH (2000) Variance of high contrast textures is sensed using negative halfwave rectification. Vision Research 40:1677-1694.

Copenhagen DR, Ashmore JF, Schnapf JK (1983) Kinetics of synaptic transmission from photoreceptors to horizontal and bipolar cells in turtle retina. Vision Research 23:363-369.

Dacey D, Petersen M (1992) Dendritic field size and morphology of midget and parasol ganglion cells of the human retina. Proceedings of the National Academy of Sciences 89:9666-9670.

Dalal SS, Zumer JM, Guggisberg AG, Trumpis M, Wong DDE, Sekihara K, Nagarajan SS (2011) MEG/EEG source reconstruction, statistical evaluation, and visualization with NUTMEG. Computational Intelligence and Neuroscience 2011:1-17.

Dalal SS, Zumer JM, Agrawal V, Hild KE, Sekihara K, Nagarajan SS (2004) NUTMEG: A neuromagnetic source reconstruction toolbox. Neurology $\&$ Clinical Neurophysiology 52.

DeVries SH, Baylor DA (1997) Mosaic arrangement of ganglion cell receptive field in rabbit retina. Journal of Neurophysiology 78:2048-2060.

Doty R, Kimura D (1963) Oscillatory potentials in the visual system of cats and monkeys. The Journal of Physiology 168:205.

Evers HU, Gouras P (1986) Three cone mechanisms in the primate electroretinogram: Two with, one without off-center bipolar responses. Vision Research 26:245-254.

Frishman LJ (2013) Retina, chapter Electrogenesis of the Electroretinogram, pp. 177-201 Saunders.

Fröhlich F (1914) Beiträge zur allgemeinen Physiologie der Sinnesorgane. Zeitschrift für Psychologie und Physiologie der Sinnesorgane 48:28-164.

Galilei G (1632) Dialogo sopra i due massimi sistemi del mondo Battista Landini, Florence, Italy.

Gollisch T, Meister M (2008) Rapid neural coding in the retina with relative spike latencies. Science 319:1108-1111.

Gramfort A, Papadopoulo T, Olivi E, Clerc M (2010) OpenMEEG: Opensource software for quasistatic bioelectromagnetics. Biomed. Eng. Online 9:45.

Gramfort A, Papadopoulo T, Olivi E, Clerc M (2011) Forward field computation with OpenMEEG. Computational Intelligence and Neuroscience 2011.

Hartline H (1938) The response of single optic nerve fibers of the vertebrate eye to illumination of the retina. American Journal of Physiology 121:400-415.

Heinrich SP, Bach M (2001) $120 \mathrm{~Hz}$ oscillations in the flash visual evoked potential are strictly phase-locked and limited to the first 100 ms. Visual Neuroscience 18:917-921.

Heinrich SP, Bach M (2004) High-frequency oscillations in human visual cortex do not mirror retinal frequencies. Neuroscience Letters 369:55-58.

Hermes D, Miller KJ, Wandell BA, Winawer J (2014) Stimulus dependence of gamma oscillations in human visual cortex. Cerebral Cortex 25:2951-2959.

Hermes D, Miller KJ, Wandell BA, Winawer J (2015) Gamma oscillations in visual cortex: the stimulus matters. Trends in Cognitive Sciences 19:57. 
Hoogenboom N, Schoffelen JM, Oostenveld R, Parkes LM, Fries P (2006) Localizing human visual gamma-band activity in frequency, time and space. NeuroImage 29:764-773.

Jin J, Weng C, Yeh CI, Gordon JA, Ruthazer ES, Stryker MP, Swadlow HA, Alonso JM (2008) On and off domains of geniculate afferents in cat primary visual cortex. Nature Neuroscience 11:88-94.

Jin JZ, Wang Y, Lashgari R, Swadlow HA, Alonso JM (2011) Faster thalamocortical processing for dark than light visual targets. Journal of Neuroscience 31:17471-17479.

Kenyon GT, Moore B, Jeffs J, Denning KS, Stephens GJ, Travis BJ, George JS, Theiler J, Marshak DW (2003) A model of high-frequency oscillatory potentials in retinal ganglion cells. Visual Neuroscience 20:465-480.

Koepsell K, Wang X, Vaingankar V, Wei Y, Wang Q, Rathbun DL, Usrey WM, Hirsch JA, Sommer FT (2009) Retinal oscillations carry visual information to cortex. Frontiers in Systems Neuroscience 3.

Komban SJ, Alonso JM, Zaidi Q (2011) Darks are processed faster than lights. Journal of Neuroscience 31:8654-8658.

Komban SJ, Kremkow J, Jin J, Wang Y, Lashgari R, Li X, Zaidi Q, Alonso JM (2014) Neuronal and perceptual differences in the temporal processing of darks and lights. Neuron 82:224-234.

Kozak WM (1971) Electroretinogram and spike activity in mammalian retina. Vision Research Supplement 3:129-149.

Krauskopf J (1980) Discrimination and detection of changes in luminance. Vision Research 20:671-677.

Kremers J, Lee BB, Pokorny J, Smith VC (1993) Responses of macaque ganglion cells and human observers to compound periodic waveforms. Vision Research 33:1997-2011.

Kremkow J, Jin J, Komban SJ, Wang Y, Lashgari R, Li K, Jansen M, Zaidi Q, Alonso JM (2014) Neuronal nonlinearity explains greater visual spatial resolution for darks than lights. Proceedings of the National Academy of Sciences 111:3170-3175.

Lankheet MJ, Lennie P, Krauskopf J (1998) Distinctive characteristics of subclasses of red-green P-cells in LGN of macaque. Visual Neuroscience 15:37-46.

Liang Z, Freed MA (2010) The ON pathway rectifies the OFF pathway of the mammalian retina. Journal of Neuroscience 30:5533-5543.

Lopez L, Sannita WG (1997) Magnetically recorded oscillatory responses to luminance stimulation in man. Electroencephalography and Clinical Neurophysiology 104:91-95.

Marmor MF, Arden GB, Nilsson SE, Zrenner E (1989) Standard for clinical electroretinography. Archives of Ophthalmology 107:816-819.

Marmor MF, Fulton AB, Holder GE, Miyake Y, Brigell M (2009) ISCEV Standard for fullfield clinical electroretinography (2008 update). Documenta Ophthalmologica 118:69-77.

Molotchnikoff S, Dubuc M, Brunette JR (1975) Simultaneous recordings of visual cortex and superior colliculus field potentials in the rabbit. Canadian Journal of Neurological Sciences/Journal Canadien des Sciences Neurologiques 2:61-66.

Morigiwa K, Tauchi M, Fukuda Y (1989) Fractal analysis of ganglion cell dendritic branching patterns of the rat and cat retinae. Neuroscience Research Supplements 10:S131-S139. 
Munk MHJ, Neuenschwander S (2000) High-frequency oscillations (20 to $120 \mathrm{~Hz}$ ) and their role in visual processing. Journal of Clinical Neurophysiology 17:341-360.

Muthukumaraswamy SD, Singh KD, Swettenham JB, Jones DK (2010) Visual gamma oscillations and evoked responses: variability, repeatability and structural MRI correlates. NeuroImage 49:3349-3357.

Nawy S, Jahr CE (1990) Suppression by glutamate of cGMP-activated conductance in retinal bipolar cells. Nature 346:269.

Neuenschwander S, Castelo-Branco M, Baron J, Singer W (2002) Feed-forward synchronization: propagation of temporal patterns along the retinothalamocortical pathway. Philosophical Transactions of the Royal Society of London B: Biological Sciences 357:1869-1876.

Nichols Z, Nirenberg S, Victor J (2013) Interacting linear and nonlinear characteristics produce population coding asymmetries between $\mathrm{ON}$ and $\mathrm{OFF}$ cells in the retina. Journal of Neuroscience 33:14958-14973.

Olman C, Boyaci H, Fang F, Doerschner K (2008) V1 responses to different types of luminance histogram contrast. Journal of Vision 8:345-345.

Oostenveld R, Fries P, Maris E, Schoffelen JM (2010) FieldTrip: open source software for advanced analysis of MEG, EEG, and invasive electrophysiological data. Computational Intelligence and Neuroscience 2011.

Perlman I (2001) Webvision: The Organization of the Retina and Visual System, chapter The Electroretinogram: ERG Online Textbook of the Visual System. University of Utah.

Saleem AB, Lien AD, Krumin M, Haider B, Rosón MR, Ayaz A, Reinhold K, Busse L, Carandini M, Harris KD (2017) Subcortical source and modulation of the narrowband gamma oscillation in mouse visual cortex. Neuron 93:315-322.

Sannita W, Conforto S, Lopez L, Narici L (1999) Synchronized 15.0-35.0 Hz oscillatory response to spatially modulated visual patterns in man. Neuroscience 89:619-623.

Sekihara K, Nagarajan SS (2008) Adaptive spatial filters for electromagnetic brain imaging Springer Science \& Business Media.

Sieving PA, Murayama K, Naarendorp F (1994) Push-pull model of the primate photopic electroretinogram: A role for hyperpolarizing neurons in shaping the b-wave. Visual Neuroscience 11:519-532.

Swettenham JB, Muthukumaraswamy SD, Singh KD (2009) Spectral properties of induced and evoked gamma oscillations in human early visual cortex to moving and stationary stimuli. Journal of Neurophysiology 102:1241-1253.

Todorov MI, Kékesi KA, Borhegyi Z, Galambos R, Gábor J, Hudetz AG (2016) Retinocortical stimulus frequency-dependent gamma coupling: Evidence and functional implications of oscillatory potentials. Physiological Reports 4.

Tzourio-Mazoyer N, Landeau B, Papathanassiou D, Crivello F, Etard O, Delcroix N, Mazoyer B, Joliot. M (2002) Automated Anatomical Labeling of activations in SPM using a macroscopic anatomical parcellation of the MNI MRI single-subject brain. NeuroImage 15:273-289.

van Pelt S, Fries P (2013) Visual stimulus eccentricity affects human gamma peak frequency. NeuroImage 78:439-447. 
bioRxiv preprint doi: https://doi.org/10.1101/153551; this version posted June 21, 2017. The copyright holder for this preprint (which was

Van Veen BD, van Drongelen W, Yuchtman M, Suzuki A (1997) Localization of brain electrical activity via linearly constrained minimum variance spatial filtering. IEEE Transaction on Biomedical Engineering 44:867-880.

Vidal JR, Chaumon M, O'Regan JK, Tallon-Baudry C (2006) Visual grouping and the focusing of attention induce gamma-band oscillations at different frequencies in human magnetoencephalogram signals. Journal of Cognitive Neuroscience 18:1850-1862.

von Helmholtz H (1867) Handbuch der physiologischen Optik, Vol. 9 Voss.

Vukmanic E, Godwin K, Shi P, Hughes A, DeMarco Jr. P (2014) Full-field electroretinogram response to increment and decrement stimuli. Documenta Ophthalmologica 129:85-95.

Wässle H, Peichl L, Boycott B (1981) Morphology and topography of on- and off-alpha cells in the cat retina. Proceedings of the Royal Society of London B: Biological Science 212:157-175.

Werblin FS, Dowling JE (1969) Organization of the retina of the mud-puppy, Necturus maculosus. II. Intracellular recording. Journal of Neurophysiology 32:339-355.

Xing D, Yeh CI, Shapley RM (2010) Generation of black-dominant responses in V1 cortex. The Journal of Neuroscience 30:13504-13512.

Yeh CI, Xing D, Shapley RM (2009) "Black" responses dominate macaque primary visual cortex V1. Journal of Neuroscience 29:11753-11760.

Zaghloul KA, Boahen K, Demb JB (2003) Different circuits for ON and OFF retinal ganglion cells cause different contrast sensitivities. Journal of Neuroscience 23:2645-2654.

Zemon V, Gordon J, Welch J (1988) Asymmetries in ON and OFF visual pathways of humans revealed using contrast-evoked cortical potentials. Visual Neuroscience 1:145-150.

Zemon V, Eisner W, Gordon J, Grose-Fifer J, Tenedios F, Shoup H (1995) Contrastdependent responses in the human visual system: childhood through adulthood. International Journal of Neuroscience 80:181-201. 